EPJ Web of Conferences 41, 10005 (2013)

DOI: $10.1051 /$ epjconf/20134110005

C Owned by the authors, published by EDP Sciences, 2013

\title{
Towards optical attosecond pulses: broadband phase coherence between an ultrafast laser and OPO using lock-to- zero CEO stabilization
}

\author{
R. A. McCracken ${ }^{1}$, J. Sun ${ }^{1,2}$, C. G. Leburn ${ }^{1}$ and D. T. Reid ${ }^{1}$ \\ ${ }^{1}$ Scottish Universities Physics Alliance (SUPA), Institute of Photonics and Quantum Sciences, \\ School of Engineering and Physical Sciences, Heriot Watt University, Riccarton, Edinburgh \\ EH14 4AS, UK \\ ${ }^{2}$ School of Physics, Huazhong University of Science and Technology, Wuhan, Hubei 430074, China
}

\begin{abstract}
The carrier-envelope-offset frequencies of the pump, signal, idler and related sum-frequency mixing pulses have been locked to $0 \mathrm{~Hz}$ in a 20-fs-Ti:sapphire-pumped optical parametric oscillator, satisfying a critical prerequisite for optical attosecond pulse synthesis.
\end{abstract}

\section{Introduction}

Nonlinear $\chi^{(2)}$ media provide frequency-conversion bandwidths of $1-2 \mathrm{PHz}$, sufficient to support sub-500-as optical fields, offering a radically different route to this temporal regime than attosecond pulses produced by high-harmonic generation. Sum-frequency-mixing (SFM) and second-harmonicgeneration (SHG) within a femtosecond optical parametric oscillator (OPO) provide a practical means of creating the short parent pulses needed to coherently synthesize sub-femtosecond pulses over a wide visible bandwidth. A major obstacle is that the parent pulses produced by these processes are normally mutually incoherent because their carrier-envelope-offset (CEO) frequencies are all different combinations of those of the pump $(p)$, signal $(s)$ and idler $(i)$ pulses. Previously, we demonstrated coherent pulse synthesis between the SHG pulses from an OPO and those of its Ti:sapphire pump laser by locking the CEO frequencies of both sources to a common value [1]. This approach can be generalized to allow pump and OPO pulses at multiple wavelengths to be made coherent by locking their CEO frequencies to $0 \mathrm{~Hz}$ [2]. Here we describe how this approach was applied to achieve broadband phase coherence between a pump laser and multiple outputs from an OPO, spanning $>0.6 \mathrm{PHz}$ in bandwidth [3].

\section{Experimental configuration and CEO stabilization of pump and OPO}

In the experimental arrangement (Figure 1) a Ti:sapphire laser producing $20-\mathrm{fs} 100-\mathrm{MHz}\left(f_{R E P}\right)$ pulses at $800 \mathrm{~nm}$ was used to synchronously pump a PPKTP OPO. The 0.5 -mm PPKTP crystal was coated on one face with a high-reflectivity NIR coating and on the other with a broadband antireflection visible-NIR coating. The OPO oscillated at $1060 \mathrm{~nm}$ and was tunable over $980 \mathrm{~nm}-$ $1200 \mathrm{~nm}$. Table 1 lists the outputs generated by non-phase-matched SHG of the $p$ and $s$ pulses and

This is an Open Access article distributed under the terms of the Creative Commons Attribution License 2.0, which permits unrestricted use, distribution, and reproduction in any medium, provided the original work is properly cited. 
SFM between the $p+s / i$ pulses. These pulses were typically observed at $\mathrm{mW}$-level average powers and output coupled through curved mirror M1.

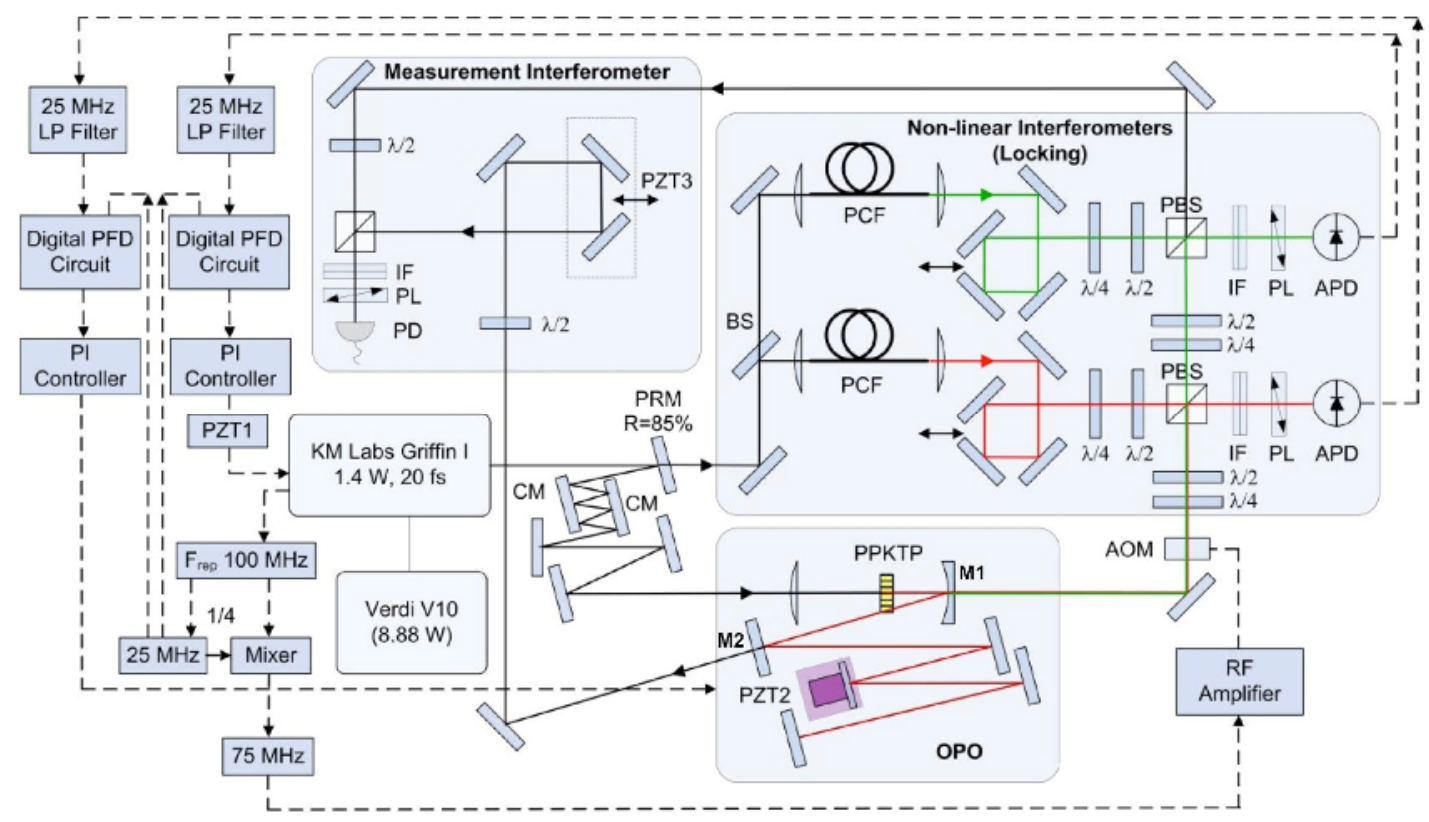

Fig. 1. Optical (solid lines) and electronic (dashed lines) layout. APD, avalanche photodide; BS, beam splitter; CM, chirped mirror; IF, interference filter; PBS, polarizing beam splitter; PD, silicon photodiode; PI, proportional integral amplifier; PL, polarizer. See text for other label definitions.

A fraction (15\%) of the pump light was used for CEO stabilization via two nonlinear interferometers (see Figure 1) in which 2 photonic crystal fibers (PCFs) generated independent pump supercontinua. The idler CEO frequency $f_{C E O}^{i}=f_{C E O}^{p}-f_{C E O}^{s}$ was obtained by interfering the 642-nm $p+i$ SFM pulses with one supercontinuum after a 10-nm bandwidth interference filter. Similarly, a beat frequency at $f_{C E O}^{p}-2 f_{C E O}^{s}$ was obtained by interfering the 530-nm SHG signal $(2 s)$ pulses with the other supercontinuum. Locking both beat frequencies to zero causes $f_{C E O}^{p}=f_{C E O}^{s}=f_{C E O}^{i}=0 \mathrm{~Hz}$, making all of the pulses listed in Table 1 mutually coherent.

Table 1. Output wavelengths from the pump and OPO.

\begin{tabular}{c|c|c|c|c|c|c|c} 
Wavelength $(\mathrm{nm})$ & 400 & 456 & 530 & 642 & 800 & 1060 & 3260 \\
\hline Origin & $2 \omega_{p}$ & $\omega_{p}+\omega_{s}$ & $2 \omega_{s}$ & $\omega_{p}+\omega_{i}$ & $\omega_{p}$ & $\omega_{s}$ & $\omega_{i}$ \\
\hline CEO frequency & $2 f_{C E O}^{p}$ & $f_{C E O}^{p}+f_{C E O}^{s}$ & $2 f_{C E O}^{s}$ & $f_{C E O}^{p}+f_{C E O}^{i}$ & $f_{C E O}^{p}$ & $f_{C E O}^{s}$ & $f_{C E O}^{i}$
\end{tabular}

CEO-locking to $0 \mathrm{~Hz}$ was achieved by blue-shifting the $p+i$ and $2 s$ pulses before the nonlinear interferometers by an acousto-optic modulator (AOM) driven at $3 f_{R E P} / 4$. The AOM can be considered to red-shift the $p+i$ and $2 s$ modes by $f_{R E P} / 4$, and for this reason we referenced the detected beat frequencies to $f_{R E P} / 4$. The resulting error signal was used to control the $p$ and $s$ CEO frequencies via 2 piezoelectric transducers (PZTs). In this way the CEO frequencies of all the pulses on the optical bench were locked to $0 \mathrm{~Hz}$. 


\section{Results and discussion}

When locked, optical heterodyning at the avalanche photodiode (APD) in each nonlinear interferometer produces a frequency at $f_{R E P}$ with sidebands at $\pm f_{R E P} / 4$. Consequently, either beat frequency can be locked to $f_{R E P} / 4$ or $3 f_{R E P} / 4$, with no electronic means of distinguishing between the two scenarios, giving a total of 4 possible locking combinations, only one of which will achieve $f_{C E O}^{p}=f_{C E O}^{s}=f_{C E O}^{i}=0$. Phase coherence was therefore confirmed by implementing a measurement interferometer (Figure 1) in which light from the second PCF, which contained a strong 530-nm component and a weaker 642-nm component, was interfered with visible light exiting one of the OPO folding mirrors. The OPO beam path was modulated using a PZT stage (PZT3) with a frequency of $1.4 \mathrm{~Hz}$ and a displacement of $400 \mu \mathrm{m}$. The beams were combined and passed through an appropriate interference filter before being detected on a silicon photodiode.

With the CEO frequencies of the pump and OPO correctly locked we observed fringes between the pump super-continuum pulses and the $p+i$ and $2 s$ pulses, indicating complete coherence (Figure 2, grey lines). When either CEO frequency was unlocked no fringes were observed, indicating a lack of coherence between the pulses (Figure 2, black lines). Observing interference at two distinct wavelengths shows that all CEO frequencies have been locked to $0 \mathrm{~Hz}$ and confirms phase-coherence across the complete ensemble of pump and OPO pulses.
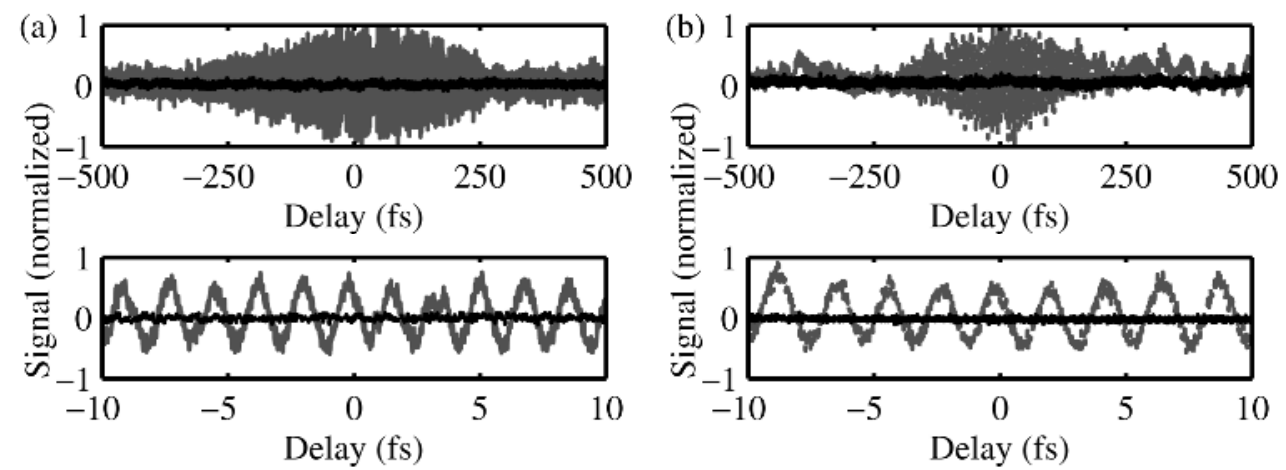

Fig. 2. Interferograms showing simultaneous phase coherence between 532-nm and 670-nm OPO outputs and a pump super-continuum. (a) Photodiode signal at $532 \mathrm{~nm}(2 s)$ with locking on (grey) and off (black); (b) photodiode signal at $670 \mathrm{~nm}(p+i)$ with locking on (grey) and off (black).

\section{Conclusions}

Phase coherence between a femtosecond OPO and its pump laser across a $0.6-\mathrm{PHz}$ bandwidth was achieved by locking the CEO frequencies of all participating pulses to $0 \mathrm{~Hz}$, confirmed by timedomain interferometry. This broadband output can be used to synthesize sub-cycle optical waveforms through direct interference of their electric fields.

\section{References}

[1] J. Sun, D.T. Reid, Opt. Lett. 34, 854 (2009)

[2] D.J. Jones, S.A. Diddams, J.K. Ranka, A. Stentz, R.S. Windeler, J.L. Hall, S.T. Cundiff, Science

28, 635 (2000).

[3] R.A. McCracken, J. Sun, C.G. Leburn, D.T. Reid, Opt. Express 20, 16269 (2012) 